\title{
Testing the lepton number of charged heavy leptons
}

\author{
Ahmed Ali \\ II. Institut für Theoretische Physik der Universität Hamburg, Hamburg, Germany \\ T. C. Yang \\ Deutsches Elektronen-Synchrotron DESY, Hamburg, Germany \\ (Received 6 April 1976)
}

\begin{abstract}
The lepton-number assignment of the charged heavy leptons (suggested by the SLAC-LBL $\mu^{ \pm} e^{\mp}$ events) has definite signatures which can be tested experimentally. We study the signatures and calculate the decay rates when the neutral currents are also contributing.
\end{abstract}

\section{INTRODUCTION}

The discovery of the "anomalous" $\mu^{ \pm} e^{\mp}$ events at SLAC ${ }^{1}$ has aroused a lot of theoretical speculation. One of the possible explanations is that such events come from the pair production of oppositely charged heavy loptons, $L^{ \pm}$, and their subsequent decays into leptons, e.g.

$$
L^{-} \rightarrow \nu_{L}+\bar{\nu}_{e(\mu)}+e^{-}\left(\mu^{-}\right)
$$

If this turns out to be the case, then one immediately asks what the lepton number is of the heavy leptons, $L^{ \pm}$- whether these heavy leptons carry the lepton numbers of $\mu^{ \pm}$and $e^{ \pm}$or a new lepton number. The question is obviously of fundamental importance. The purpose of this note is to study this question.

First we remark that if $L^{-}\left(L^{+}\right)$has the same lepton number as $\mu^{-}\left(\mu^{+}\right)$, it could be produced by the $\nu_{\mu}\left(\bar{\nu}_{\mu}\right)$ beams available at Fermilab and CERN. Albright has argued from present neutrino data that it is unlikely to be the candidate for the "anomalous" $\mu e$ events in $e^{+} e^{-}$annihilation. ${ }^{2}$ Moreover, the mass of negatively charged muontype heavy lepton is set experimentally above $8 \mathrm{GeV} .^{3}$ Since no $\nu_{e}\left(\bar{\nu}_{e}\right)$ experiment is presently available, no such experimental constraint is imposed if $L^{ \pm}$has the electronic lepton number. ${ }^{4}$ We shall concentrate below on the difference between charged heavy leptons, $L^{ \pm}$, carrying the electronic lepton number and those carrying a new lepton number. (The latter case is usually referred to as a sequential heavy lepton.)

If $L^{ \pm}$has the electronic lepton number, ${ }^{5}$ one has two alternative assignments: $L^{-}$has the lepton number as either $e^{+}$or $e^{-}$with the respective decays

$$
\begin{aligned}
& L^{-} \rightarrow \bar{\nu}_{L}+e^{-}+\bar{\nu}_{e}, \\
& L^{-} \rightarrow \nu_{L}+e^{-}+\bar{\nu}_{e} .
\end{aligned}
$$

In the first case, one finds by Fermi statistics

$$
\Gamma\left(L^{-} \rightarrow \bar{\nu}_{L}+\bar{\nu}_{e}+e^{-}\right)=2 \Gamma\left(L^{-} \rightarrow \bar{\nu}_{L}+\bar{\nu}_{\mu}+\mu^{-}\right) .
$$

It follows then that

$$
\sigma\left(e^{+} e^{-}\right): \sigma\left(\mu^{+} e^{-}+\mu^{-} e^{+}\right): \sigma\left(\mu^{+} \mu^{-}\right):: 4: 4: 1,
$$

where

$$
\sigma\left(l_{1} l_{2}\right)=\sigma\left(e^{+} e^{-} \rightarrow L^{+} L^{-}-l_{1} l_{2}+\cdots\right) .
$$

The present SLAC data are consistent with the above ratios being $1: 2: 1{ }^{1}$ The data indicate that the $L^{ \pm}$. do not carry the $e^{\mp}$ lepton numbers. Thus the possibilities left are the following: either $L^{ \pm}$have the same quantum number as $e^{ \pm}$or else they carry a new lepton number. If the latter is true, there exists then a new neutrino (massive or massless). If the former is true, one may or may not have a new neutrino. We devise tests to distinguish between these two assignments. The tests essentially consist of measuring the transitions $L^{ \pm} \rightarrow e^{ \pm}+X$, where $X$ can be either lepton(s) or hadron(s). We shall also comment on how to distinguish such decays from the heavyhadron decays, which can also simulate the heavylepton decays.

The paper is organized as follows: In Sec II we discuss the qualitative features of the two $L^{ \pm}$ lepton-number assignments. These properties are testable experimentally and should be looked for. In Sec. III we calculate starting from a phenomenological Lagrangian all the leptonic and semileptonic decays of $L^{ \pm}$involving nondiagonal neutral-current transitions $L^{ \pm} \rightarrow e^{ \pm}+X$. Section IV contains remarks.

\section{EXPERIMENTAL TESTS FOR THE LEPTON NUMBER OF CHARGED HEAVY LEPTONS}

If $L^{-}$has the quantum number of $e^{-}$, then, in addition to the charged-current decay modes

$$
\begin{aligned}
& L^{-} \rightarrow \nu_{L}+\bar{\nu}_{e}+e^{-}, \quad \nu_{L}+\bar{\nu}_{\mu}+\mu^{-}, \\
& L^{-} \rightarrow \nu_{L}+\text { hadrons },
\end{aligned}
$$


it can also decay via neutral-current modes,

$$
\begin{aligned}
& L^{-} \rightarrow e^{-}+e^{+} e^{-}, e^{-}+\mu^{+} \mu^{-}, e^{-}+\nu_{e} \bar{\nu}_{e}, \\
& \qquad e^{-}+\nu_{\mu} \bar{\nu}_{\mu}, e^{-}+\nu_{L} \bar{\nu}_{L}, \\
& L^{-} \rightarrow e^{-}+\text {hadrons, }
\end{aligned}
$$

where in (2.3) (see Fig. 1) $\nu_{L}=\nu_{e}$ if $m\left(\nu_{L}\right)=0$, otherwise it is a different particle with the same lepton number as $\nu_{e}$. The decay rates and branching ratios for (2.1) and (2.2), assuming only charged currents, already exist in literature ${ }^{6}$ but will have to be modified if nondiagonal neutral currents are present. These modifications together with the decay rates for (2.3) and (2.4) are presented in Sec III.

If $L^{-}$is a sequential heavy lepton, then only (2.1) and (2.2) are possible decay modes ${ }^{7}$ with $\nu_{L} \neq \nu_{e}$, even if $m\left(\nu_{L}\right)=0$. In principle, the mass of the neutral heavy lepton, $\nu_{L}$, can be measured by the cross section and momentum distributions of $e^{+} e^{-} \rightarrow L^{+} L^{-} \rightarrow \mu^{ \pm} e^{\mp}+$ neutrinos.

The nondiagonal neutral-current processes will have one of the following signatures:

(1) Events of the type $e^{+} e^{-} \rightarrow \mu^{ \pm} e^{\mp}+$ hadrons + missing momentum ${ }^{8}$ cannot come from the decays of sequential heavy leptons. They can arise either by heavy-lepton decays involving (2.3) and (2.4) or by leptonic and semileptonic decays of heavy hadrons. These two mechanisms can be disentangled by looking at the energy-momentum distribution of the final states. (For example, $\mu^{\mp}$ from $L^{\mp}$ decay must follow three-body decay.) One should particularly look for the processes

$$
e^{+} e^{-} \rightarrow \mu^{ \pm} e^{\mp} \pi^{0}, \mu^{ \pm} e^{\mp} \rho^{0}, \mu^{ \pm} e^{\mp} \omega^{0}, \mu^{ \pm} e^{\mp} \eta, \ldots \text {. }
$$

In the case of a single hadron in the final states, the $e^{\mp}$ and the hadron must have a unique invariant mass equal to $m_{L}$ if due to $L^{\mp}$ decay and will not do so if due to new hadron decay-in the latter case, the hadron follows three-body decay (see Fig. 2). Thus these two cases can be distin-
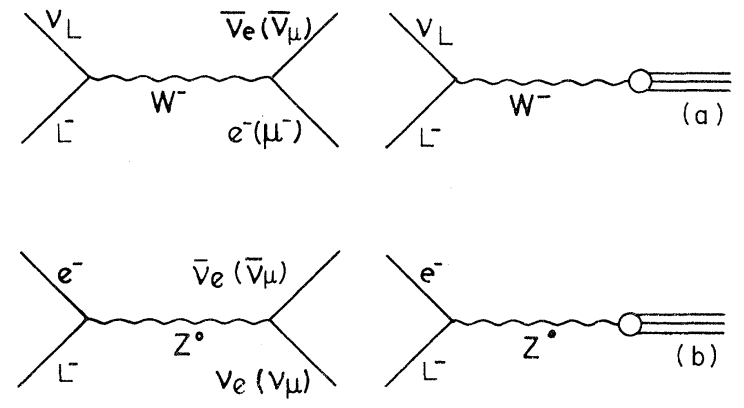

FIG. 1. (a) charged-current contribution to $L^{-}$decays; (b) neutral-current contribution to $L^{-}$decays.

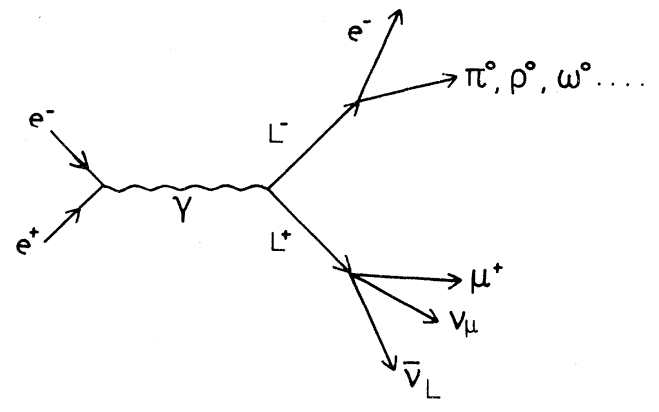

(a)

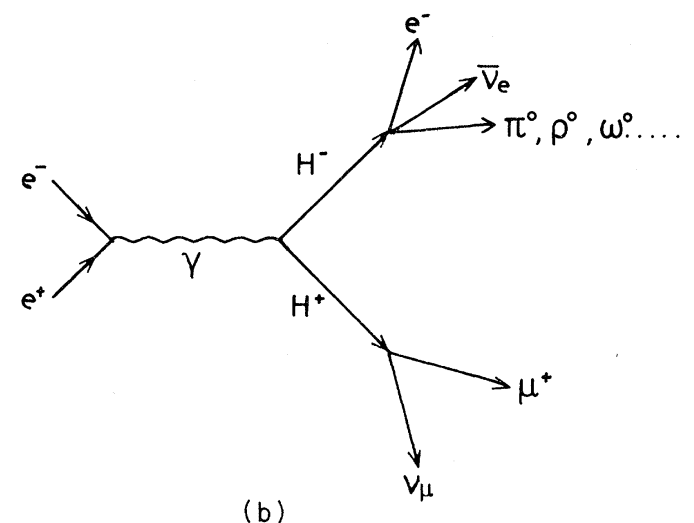

(b)

FIG. 2. Schematic diagrams for $e^{+} e^{-} \rightarrow e^{ \pm} \mu^{\mp}+$ missing momentum $+\pi^{0}, \rho 0, \omega, \ldots$ due to (a) charged-heavylepton pair decays and (b) charged-hadron pair decays.

guished.

(2) If $L$ carrys electronic lepton number, then the branching ratio for $L^{-} \rightarrow \mu^{-}+\bar{\nu}_{\mu}+\nu_{L}$ and $L^{-} \rightarrow e^{-}+\bar{\nu}_{e}+\nu_{L}$ could differ owing to the neutralcurrent contribution. Thus (a small) deviation in the ratio of anomalous $e^{+} e^{-}$and $\mu^{+} \mu^{-}$cross sections from one could indicate a nonzero neutral-current contribution.

(3) For four-charged-lepton production in $e^{+} e^{-}$ annihilation, observation of the following processes and narrow $e^{-} e^{+} e^{-}$resonance in

$$
e^{+} e^{-} \rightarrow \mu^{ \pm} e^{\mp}+e^{+} e^{-}\left(\mu^{+} \mu^{-}\right)+\text {missing momentum }
$$

of order $G_{F}^{2}$ will prove that $L^{-}$has the same lepton number as $e^{-}$. Moreover, $\sigma\left(\mu^{ \pm} e^{\mp}+e^{+} e^{-}\right)=2 \sigma\left(\mu^{ \pm} e^{\mp}\right.$ $\left.+\mu^{+} \mu^{-}\right)$. Let us give a word of caution: The strengths of leptonic decays (2.3) and semileptonic decays (2.4) are a priori unrelated; thus it is necessary to check both (1) and (3) experimentally.

In conclusion, in order to establish that the charged heavy lepton is sequential, one must check that the signatures (1)-(3) are not found. On the other hand, a clean signature (even one or few events) of (1)-(3) would prove that $L$ is a non- 
sequential charged heavy lepton. In the latter case, one must further check the following:

(4) If $L$ carrys electronic number, no processes of the kind $e^{+} e^{-} \rightarrow \mu^{+} \mu^{-}+$hadrons + missing momentum are allowed.

\section{NEUTRAL-CURRENT CONTRIBUTION TO HEAVY-CHARGED-LEPTON DECAYS}

We start with the following weak-interaction Hamiltonian:

$$
H_{\mathrm{eff}}=\frac{G_{F}}{\sqrt{2}}\left[J^{W, \mu}(x) J_{\mu}^{+W}(0)+J^{Z, \mu}(x) J_{\mu}^{+Z}\right],
$$

where $G_{F}=1.05 \times 10^{-5} \mathrm{~m}_{p}^{-2}$ is the Fermi coupling constant. $J_{\mu}^{W}$ and $J_{\mu}^{Z}$ are the charged and neutral weak currents respectively. The charged currents are defined by

$$
\begin{aligned}
J_{\mu}^{W, \text { leptonic }} & =\bar{\nu}_{e} \gamma_{\mu}\left(1-\gamma_{5}\right) e+\bar{\nu}_{\mu} \gamma_{\mu}\left(1-\gamma_{5}\right) \mu \\
& +\bar{\nu}_{L} \gamma_{\mu}\left[g_{L}^{\frac{1}{2}}\left(1-\gamma_{5}\right)+g_{R}^{\frac{1}{2}}\left(1+\gamma_{5}\right)\right] L,
\end{aligned}
$$

$$
J_{\mu}^{W, \text { hadronic }}=\left(V_{\mu}^{1 \pm i 2}-A_{\mu}^{1 \pm i 2}\right) \cos \theta+\left(V_{\mu}^{4 \pm i 5}-A_{\mu}^{4 \pm i 5}\right) \sin \theta
$$

The neutral weak currents are parametrized as follows:

$$
\begin{aligned}
& J_{\mu}^{Z \text {, leptonic }}=\alpha \bar{\nu}_{e} \gamma_{\mu} \frac{1}{2}\left(1-\gamma_{5}\right) \nu_{e}+\beta \bar{\nu}_{L} \gamma_{\mu} \frac{1}{2}\left(1-\gamma_{5}\right) \nu_{e} \\
& +\bar{e} \gamma_{\mu}\left[a_{L} \frac{1}{2}\left(1-\gamma_{5}\right)+a_{R}^{\left.\frac{1}{2}\left(1+\gamma_{5}\right)\right] e}\right. \\
& +\bar{e} \gamma_{\mu}\left[b_{L} \frac{1}{2}\left(1-\gamma_{5}\right)+b_{R} \frac{1}{2}\left(1+\gamma_{5}\right)\right] L+\cdots \\
& + \text { H.c. }+\left\{e \rightarrow \mu, L \rightarrow M, \nu_{e, L} \rightarrow \nu_{\mu, M}\right\}
\end{aligned}
$$

and

$$
\begin{aligned}
J_{\mu}^{Z, \text { hadronic }} & =h_{3} V_{\mu}^{(3)}+h_{8} V_{\mu}^{(8)}+h_{0} V_{\mu}^{(0)} \\
& +k_{3} A_{\mu}^{(3)}+k_{8} A_{\mu}^{(8)}+\cdots,
\end{aligned}
$$

where the superscripts refer to the SU(3) transformation property of the hadronic currents. The leptonic widths are [neglecting $O\left(m_{e} / m_{L}\right)$ terms]

$$
\begin{aligned}
& \frac{\Gamma\left(L^{-} \rightarrow e^{-}+\bar{\nu}_{e}+\nu_{L}\right)}{\Gamma\left(\mu^{-}-\nu_{\mu}+e^{-}+\bar{\nu}_{e}\right)}=\left(\frac{m_{L}}{m_{\mu}}\right)^{5}\left\{\left[\frac{1}{4}\left(\left|g_{L}\right|^{2}+\left|g_{R}\right|^{2}\right)+|\beta|^{2 \frac{1}{4}}\left(\left|b_{L}\right|^{2}+\left|b_{R}\right|^{2}\right)\right] f_{1}(Z)+2 \operatorname{Re} \frac{1}{4}\left(g_{R}^{*} g_{L}\right) f_{2}(Z)\right\} \\
& \frac{\Gamma\left(L^{-} \rightarrow e^{-}+e^{+} e^{-}\right)}{\Gamma\left(\mu^{-}-\nu_{\mu}+e^{-}+\bar{\nu}_{e}\right.}=\frac{1}{4}\left(\frac{m_{L}}{m_{\mu}}\right)^{5}\left(\left|a_{L}\right|^{2}+\left|a_{R}\right|^{2}\right)\left(\left|b_{L}\right|^{2}+\left|b_{R}\right|^{2}\right) .
\end{aligned}
$$

The expression for $L^{-} \rightarrow e^{-} \mu^{+} \mu^{-}$and $L^{-} \rightarrow \mu^{-} \bar{\nu}_{\mu} \nu_{L}$ can be written immediately from the above two equations, neglecting $O\left(m_{\mu} / m_{L}\right)$ terms. Fermi statistics then gives

$$
2 \Gamma\left(L^{-} \rightarrow e^{-}+\mu^{+} \mu^{-}\right)=\Gamma\left(L^{-} \rightarrow e^{-}+e^{+} e^{-}\right),
$$

and the absence of neutral-current contribution leads to

$$
\frac{\Gamma\left(L^{-}-\mu^{-} \bar{\nu}_{\mu} \nu_{L}\right)}{\Gamma\left(\mu^{-}-\nu_{\mu} e^{-} \bar{\nu}_{e}\right)}=\left(\frac{m_{L}}{m_{\mu}}\right)^{5}\left[\frac{1}{4}\left(\left|g_{L}\right|^{2}+\left|g_{R}\right|^{2}\right) f_{1}(Z)+2 \operatorname{Re}\left(\frac{1}{4}\left(g_{R}^{*} g_{L}\right)\right) f_{2}(Z)\right]
$$

where $Z=m\left(\nu_{L}\right) / m(L)$ and

$$
\begin{aligned}
& f_{1}(Z)=\left(1-Z^{4}\right)\left(Z^{4}-8 Z^{2}+1\right)+24 Z^{4} \ln \left(\frac{1}{Z}\right) . \\
& f_{2}(Z)=4 Z\left(1-Z^{2}\right)^{3}-6 Z\left(1+Z^{2}\right)\left[1-Z-4 Z^{2} \ln \left(\frac{1}{Z}\right)\right],
\end{aligned}
$$

The neglect of $O\left(m_{\mu} / m_{L}\right)$ terms does not introduce any appreciable effect if $m_{L}>1.5 \mathrm{GeV}$.

\section{A. Hadronic decay modes}

Defining the spectral functions $\rho_{1}^{W}$ and $\rho_{2}^{W}$ for the charged weak currents and $\rho_{1}^{Z}$, and $\rho_{2}^{Z}$ for the neutral weak currents by

$$
\begin{aligned}
\sum_{F}\left\langle 0\left|J_{\mu(0)}^{W(Z)}\right|\right. & F\rangle\left\langle F\left|J_{\nu(0)}^{+W(Z)}\right| 0\right\rangle \delta^{4}\left(q-p_{F}\right)(2 \pi)^{3} \\
& \equiv \rho_{1}^{W(Z)}\left(q^{2}\right)\left(q_{\mu} q_{\nu}-q^{2} g_{\mu \nu}\right)+\rho_{2}^{W(Z)}\left(q^{2}\right) q_{\mu} q_{\nu},
\end{aligned}
$$

we obtain, if the hadrons have invariant mass $\sqrt{t}$, $\frac{d \Gamma}{d t}\left(L^{ \pm} \rightarrow e^{ \pm}+\right.$hadrons $)$

$$
=\frac{G_{F}^{2} m_{L}^{3}}{16 \pi}\left(1-\frac{t}{m_{L}^{2}}\right)\left(\frac{\left|b_{L}\right|^{2}+\left|b_{R}\right|^{2}}{4}\right) g_{1}^{Z}(t) \text {, }
$$

where 


$$
g_{1}^{Z}(t)=\rho_{1}^{Z}(t)\left(1+\frac{t}{m_{L}{ }^{2}}-\frac{2 t^{2}}{m_{L}^{4}}\right)+\rho_{2}^{Z}(t)\left(1-\frac{t}{m_{L}^{2}}\right)
$$

The semileptonic decays of $L^{ \pm}$, involving charged weak currents, have been studied in detail in the literature, ${ }^{6}$ and we shall not discuss them here. The hadronic continuum contribution and the single-particle contribution involving the neutral current can be calculated in an analogous way and are given below.

\section{B. Hadronic continuum contribution}

To estimate the hadronic continuum contribution, we shall invoke the notion of asymptotic chiral symmetry ${ }^{9}$ and asymptotic $\mathrm{U}(3)$ symmetry, ${ }^{10}$ which is probably a reasonable assumption when $t$ is large $\left(t>1 \mathrm{GeV}^{2}\right)$. Expressing $\rho_{1,2}^{Z}(t)$ in terms of the parameters introduced through Eq. (3.4),

$$
\begin{aligned}
\rho_{1}^{Z}(t)= & \left|h_{3}\right|^{2} \rho_{1 V}^{33}(t)+\left|h_{8}\right|^{2} \rho_{1 V}^{88}(t)+\left|h_{0}\right|^{2} \rho_{1 V}^{00}(t) \\
& +\left|k_{3}\right|^{2} \rho_{1 A}^{33}(t)+\left|k_{8}\right|^{2} \rho_{1 A}^{88}(t), \\
\rho_{2}^{Z}(t)= & \left|k_{3}\right|^{2} \rho_{2 A}^{33}(t)+\left|k_{8}\right|^{2} \rho_{2 A}^{88}(t),
\end{aligned}
$$

the asymptotic chiral $\mathrm{SU}(3) \times \mathrm{SU}(3)$ symmetry can now be formulated as follows:

$$
\begin{aligned}
& \lim _{t \rightarrow \infty} \rho_{2 A}^{i j}(t)=0, \quad i, j=3,8 \\
& \lim _{t \rightarrow \infty} \rho_{1 A}^{i i}(t)=\lim _{t \rightarrow \infty} \rho_{1 V}^{i i}(t) . \quad i, j=3,8
\end{aligned}
$$

With asymptotic $\mathrm{U}(3)$ symmetry, spectral functions of different $\mathrm{U}(3)$ components are related (to the spectral function of electromagnetic currents), and one has

$$
\begin{aligned}
\lim _{t \rightarrow \infty} \rho_{1 V}^{00}(t) & =\lim _{t \rightarrow \infty} \rho_{1 V}^{88}(t) \\
& =\lim _{t \rightarrow \infty} \rho_{1 V}^{33}(t) \\
& =\frac{1}{8 \pi^{2}} \lim _{s \rightarrow \infty} \frac{\sigma_{e^{+} e^{-}-\text {hadrons }}^{\prime}}{\sigma_{e^{+} e^{-} \rightarrow \mu^{+} \mu^{-}}},
\end{aligned}
$$

where $\sigma_{e^{+} e^{-} \rightarrow \text { hadrons }}^{\prime}$ denotes the hadronic total cross section excluding the heavy-lepton contribution. We get [using (3.10)-(3.17)]

$\Gamma\left(L^{-} \rightarrow e^{-}+\right.$hadron continuum $)$

$$
\begin{aligned}
& \Gamma\left(L^{-} \rightarrow \mu^{-}+\bar{\nu}_{\mu}+\nu_{L}\right) \\
& =\frac{3}{4} h C R\left(\frac{\Lambda^{2}}{m_{L}^{2}}\right) \\
& \quad \times\left[\frac{\left|b_{L}\right|^{2}+\left|b_{R}\right|^{2}}{\left(\left|g_{L}\right|^{2}+\left|g_{R}\right|^{2}\right) f_{1}(Z)+2 \operatorname{Re}\left(g_{R}^{*} g_{L}\right) f_{2}(Z)}\right],
\end{aligned}
$$

where

$$
\begin{aligned}
& C=\lim _{s \rightarrow \infty} \frac{\sigma_{e^{+} e^{-\rightarrow h a d r o n s}}^{\prime}}{\sigma_{e^{+} e^{-\rightarrow \mu^{+} \mu^{-}}}}=\text {constant } \\
& h=\left|h_{0}\right|^{2}+\left|h_{3}\right|^{2}+\left|h_{8}\right|^{2}+\left|k_{3}\right|^{2}+\left|k_{8}\right|^{2},
\end{aligned}
$$

and

$$
R\left(\frac{\Lambda^{2}}{m_{L}{ }^{2}}\right)=1-\frac{\Lambda^{2}}{2 m_{L}{ }^{2}}+\frac{\Lambda^{6}}{2 m_{L}{ }^{6}}-\frac{\Lambda^{8}}{m_{L}{ }^{8}},
$$

where $\Lambda$ is the threshold for estimating the hadron continuum (usually taken to be $\sim 1 \mathrm{GeV}$ ). For comparison, the charged-current contribution to the hadron continuum is given by

$$
\frac{\Gamma\left(L^{-}-\nu_{L}+\text { hadronic continuum }\right)}{\Gamma\left(L^{-} \rightarrow \nu_{L}+\mu^{-}+\bar{\nu}_{\mu}\right)}=\frac{3}{2} C R\left(\Lambda^{2} / m_{L}^{2}\right) \text {. }
$$

\section{Single-particle contributions}

The various single-particle contributions can be obtained from (3.11) by using the following pole-dominated forms of the various spectral functions. (The superscript refers to the intermediate state saturating the spectral function.) The $\rho^{\alpha \beta}(\alpha, \beta=0,8)$ are evaluated by assuming the usual SU(3) breaking;

$$
\begin{aligned}
& \rho_{1}^{\pi}=\rho_{1}^{\eta}=\rho_{1}^{\eta^{\prime}}=0, \\
& \rho_{2}^{\pi}=\frac{1}{2} F_{\pi}{ }^{2} \delta\left(t-m_{\pi}{ }^{2}\right) .
\end{aligned}
$$

The formula for $\rho_{2}^{\eta}$ and $\rho_{2}^{\eta \prime}$ is similar, with $F_{\pi} \rightarrow F_{\eta}, m_{\pi} \rightarrow m_{\eta}$, etc. :

$$
\begin{aligned}
& \rho_{1 V}^{\rho}=\frac{m_{\rho}^{2}}{f_{\rho}} \delta\left(t-m_{\rho}^{2}\right), \\
& \rho_{1 V}^{88, \omega}=\frac{3}{4}\left(m_{\omega}^{2} f_{y}^{-1} \sin \theta_{y}\right)^{2} \delta\left(t-m_{\omega}{ }^{2}\right), \\
& \rho_{1 V}^{00, \omega}=\frac{3}{2}\left(m_{\omega}^{2} f_{B}^{-1} \cos \theta_{B}\right)^{2} \delta\left(t-m_{\omega}^{2}\right), \\
& \rho_{1 V}^{08, \omega}=\frac{3}{2 \sqrt{2}}\left(m_{\omega}^{2} f_{B}^{-1} \cos \theta_{B}\right)\left(m_{\omega}^{2} f_{y}^{-1} \sin \theta_{y}\right) \delta\left(t-m_{\omega}{ }^{2}\right), \\
& \rho_{1 V}^{88, \phi}=\frac{3}{4}\left(m_{\varphi}^{2} f_{y}^{-1} \cos \theta_{y}\right)^{2} \delta\left(t-m_{\varphi}^{2}\right), \\
& \rho_{1 V}^{00, \phi}=\frac{3}{2}\left(m_{\varphi}^{2} f_{B}^{-1} \sin \theta_{B}\right)^{2} \delta\left(t-m_{\varphi}^{2}\right), \\
& \rho_{1 V}^{08, \phi}=\frac{3}{2 \sqrt{2}}\left(m_{\varphi}^{2} f_{y}{ }^{-1} \cos \theta_{y}\right)\left(m_{\varphi}^{2} f_{B}^{-1} \sin \theta_{B}\right) \delta\left(t-m_{\varphi}{ }^{2}\right) .
\end{aligned}
$$

We refer to Ref. 11 for the definition of various coupling constants and mixing parameters used in Eq. (3.23). The decay rates using (3.23) are listed in Table I, where we have normalized with respect to the charged-weak-current process $L^{ \pm} \rightarrow \pi^{ \pm}+\bar{\nu}_{L}\left(\nu_{L}\right)$; 
TABLE I. Two-body decay rates of charged heavy leptons by neutral weak currents. $\theta_{B}, \theta_{y}$, and $f_{B}, f_{y}$ are, respectively, the $\omega-\phi$ mixing angles and coupling constants introduced in Ref. 11.

$$
\begin{aligned}
& \frac{\Gamma\left(L^{ \pm} \rightarrow e^{ \pm}+\pi^{\theta}\right)}{\Gamma\left(L^{-} \rightarrow \nu+\pi^{-}\right)}=\frac{1}{2} \delta\left|k_{3}\right|^{2} \\
& \frac{\Gamma\left(L^{ \pm} \rightarrow e^{ \pm}+\eta\right)}{\Gamma\left(L^{-} \rightarrow \nu+\pi^{-}\right)}=\frac{1}{2} \delta\left|k_{8}\right|^{2} \frac{F_{\eta}^{2}}{F_{\pi}^{2}}\left(1-\frac{m \eta^{2}}{m_{L^{2}}}\right)^{2} \\
& \frac{\Gamma\left(L^{ \pm} \rightarrow e^{ \pm}+\eta^{\prime}\right)}{\Gamma\left(L^{-} \rightarrow \nu+\pi^{-}\right)}=\frac{1}{2} \delta\left|k_{8}\right|^{2} \frac{F_{\eta^{\prime}}{ }^{2}}{F_{\pi}^{2}}\left(1-\frac{m_{\eta^{\prime}}{ }^{2}}{m_{L}{ }^{2}}\right)^{2} \\
& \frac{\Gamma\left(L^{ \pm} \rightarrow e^{ \pm}+\rho^{0}\right)}{\Gamma\left(L^{-} \rightarrow \nu+\pi^{-}\right)}=\delta\left|h_{3}\right|^{2}\left(\frac{m_{\rho}^{2}}{F_{\pi} F_{\rho}}\right)^{2}\left(1-\frac{m_{\rho}^{2}}{m_{L}^{2}}\right)^{2}\left(1+\frac{2 m_{\rho}^{2}}{m_{L}^{2}}\right) \\
& \frac{\Gamma\left(L^{ \pm} \rightarrow e^{ \pm}+\omega\right)}{\Gamma\left(L^{-} \rightarrow \nu+\pi^{-}\right)}=\delta\left(\frac{m_{\omega^{2}}^{2}}{F_{\pi}}\right)^{2}\left(1-\frac{m_{\omega^{2}}{ }^{2}}{m_{L}{ }^{2}}\right)^{2}\left(1+\frac{2 m_{\omega^{2}}{ }^{2}}{m_{L}{ }^{2}}\right)\left[\left|h_{8}\right|^{2} \frac{\sin \theta_{y}}{f_{y}^{2}}+\left|h_{0}\right|^{2} \frac{\cos ^{2} \theta_{B}}{f_{B}{ }^{2}}+2 \operatorname{Re}\left(h_{8}^{*} h_{0}\right) \frac{\sin \theta_{y} \cos \theta_{B}}{f_{y} f_{B}}\right] \\
& \frac{\Gamma\left(L^{ \pm} \rightarrow e^{ \pm}+\omega\right)}{\Gamma\left(L^{-} \rightarrow \nu+\pi^{-}\right)}=\delta\left(\frac{m_{\varphi}^{2}}{F_{\pi}}\right)^{2}\left(1-\frac{m \varphi^{2}}{m_{L^{2}}{ }^{2}}\right)^{2}\left(1+\frac{2 m \varphi^{2}}{m_{L}{ }^{2}}\right)\left[\left|h_{8}\right|^{2} \frac{\cos ^{2} \theta_{y}}{f_{y}{ }^{2}}+\left|h_{0}\right|^{2} \frac{\sin ^{2} \theta_{B}}{f_{B}{ }^{2}}+2 \operatorname{Re}\left(h_{8}^{*} h_{0}\right) \frac{\cos \theta_{y} \sin \theta_{B}}{f_{B} f_{y}}\right] \\
& \frac{\Gamma\left(L^{ \pm} \rightarrow e^{ \pm}+A_{1}\right)}{\Gamma\left(L^{-} \rightarrow \nu+\pi^{-}\right)}=\delta\left|k_{3}\right|^{2}\left(\frac{m_{A_{1}}{ }^{2}}{f_{A_{1}} F_{\pi}}\right)^{2}\left(1-\frac{m_{A_{1}}{ }^{2}}{m_{L^{2}}}\right)\left(1+\frac{2 m_{A_{1}}{ }^{2}}{m_{L}{ }^{2}}\right)
\end{aligned}
$$

where

$\delta=\left(\frac{\left|b_{L}\right|^{2}+\left|b_{R}\right|^{2}}{\left|g_{L}\right|^{2}+\left|g_{R}\right|^{2}}\right)$

$$
\begin{aligned}
\Gamma\left[L^{ \pm} \rightarrow \pi^{ \pm}+\bar{\nu}_{L}\left(\nu_{L}\right)\right] & =\frac{G_{F}^{2} m_{L}^{3} F_{\pi}^{2}}{16 \pi}\left[\frac{1}{4}\left(\left|g_{L}\right|^{2}+\left|g_{R}\right|^{2}\right) f_{1}(Z)+2 \operatorname{Re}\left(\frac{1}{4}\left(g_{R}^{*} g_{L}\right)\right) f_{2}(Z)\right] \\
& \simeq 3 \times 10^{11} \mathrm{sec}^{-1} \text { for } g_{L}=1, g_{R}=0, m_{L}=1.8 \mathrm{GeV}, m_{\nu_{L}}=0 .
\end{aligned}
$$

Terms of order $m_{\pi}{ }^{2} / m_{L}{ }^{2}$ and $m_{l}{ }^{2} / m_{L}{ }^{2}$ are neglected in all the equations. $Z$ and $f_{1,2}(Z)$ are defined previously.

\section{Leptonic branching ratios}

For practical purposes we have calculated the leptonic branching ratios as follows (setting $\left.m_{\nu_{L}}=0\right)$ :

$$
\begin{aligned}
& \frac{\Gamma\left(L^{-}-\mu^{-} \bar{\nu}_{\mu} \nu_{e}\right)}{\Gamma_{\text {total }}}=\frac{1}{2+\delta|\beta|^{2}+\frac{3}{2} \delta \gamma+x+\delta y}, \\
& \frac{\Gamma\left(L^{-}-e^{-} \bar{\nu}_{e} \nu_{L}\right)}{\Gamma_{\text {total }}}=\frac{1+\delta|\beta|^{2}}{2+\delta|\beta|^{2}+\frac{3}{2} \delta \gamma+x+\delta y}, \\
& \frac{\Gamma\left(L^{-}-e^{-} e^{+} e^{-}\right)}{\Gamma_{\text {total }}}=\frac{\delta \gamma}{2+\delta|\beta|^{2}+\frac{3}{2} \delta \gamma+x+\delta y}, \\
& \Gamma\left(L^{-} \rightarrow e^{-} \mu^{+} \mu^{-}\right)=\frac{1}{2} \Gamma\left(L^{-}-e^{-} e^{+} e^{-}\right),
\end{aligned}
$$

where

$$
\begin{aligned}
& \delta=\frac{\left|b_{L}\right|^{2}+\left|b_{R}\right|^{2}}{\left|g_{L}\right|^{2}+\left|g_{R}\right|^{2}}, \\
& \gamma=\left|a_{L}\right|^{2}+\left|a_{R}\right|^{2},
\end{aligned}
$$

$$
\begin{aligned}
& x=\frac{12 \pi^{2}}{m_{L}^{2}} \int_{0}^{m_{L} L^{2}}\left(1-\frac{t}{m_{L}^{2}}\right) g_{1}^{W}(t), \\
& y=\frac{12 \pi^{2}}{m_{L}{ }^{2}} \int_{0}^{m_{L}{ }^{2}}\left(1-\frac{t}{m_{L}^{2}}\right) g_{1}^{z}(t),
\end{aligned}
$$

and

$$
g_{1}^{W}(t)=\rho_{1}^{W}(t)\left(1+\frac{t}{m_{L}^{2}}-\frac{2 t^{2}}{m_{L}^{4}}\right) .
$$

$\rho_{1,2}^{W, Z}(t)$ are the spectral functions defined through (3.10). We emphasize that the parameter $\gamma$ which enters in the weak contribution to the process $e^{+} e^{-} \rightarrow e^{+} e^{-}$can be, in principle, very different owing to the different masses of the heavy bosons mediating $e^{+} e^{-} \rightarrow e^{+} e^{-}$and $L^{ \pm} \rightarrow e^{ \pm} e^{+} e^{-}$.

\section{REMARKS}

After discussing methods to test the quantum numbers of the new charged heavy lepton and calculating the decay rates in previous sections, we list in Table II some possible lepton schemes. ${ }^{12-14}$ Definite features can be used to rule out or dis- 
TABLE II. Classifications of lepton models.

\begin{tabular}{|c|c|c|}
\hline \multirow{2}{*}{$\begin{array}{l}\text { Lepton } \\
\text { representations }\end{array}$} & \multicolumn{2}{|c|}{ Heavy leptons } \\
\hline & Charged & Neutral \\
\hline Doublets $=\mathrm{SU}(2) \times \mathrm{U}(1)$ models $^{12}$ & $\begin{array}{l}\text { Most models have only one sequential } \\
\text { charged heavy lepton, produced } \\
\text { only in } e^{+} e^{-} \text {annihilation }\end{array}$ & $\begin{array}{l}\text { One or more, but not produced by ordinary } \\
\text { neutrino scattering in most models }\end{array}$ \\
\hline $\begin{array}{l}\text { Triplets: (a) Three triplets } \\
\text { with Han-Nambu } \\
\text { charge struc- } \\
\text { ture }^{13}\end{array}$ & $\begin{array}{l}\text { Two sequential charged heavy leptons } \\
\text { produced in } e^{+} e^{-} \text {annihilation }\end{array}$ & $\begin{array}{l}\text { One produced by } \nu_{\mu} \text { scattering and one } \\
\text { produced by } \nu_{e} \text { scattering }\end{array}$ \\
\hline $\begin{array}{c}\text { Triplets: (b) Arbitrary charge } \\
\text { structure }^{14}\end{array}$ & Flexible & Flexible \\
\hline $\begin{array}{l}\text { Quadruplets with electron and } \\
\text { muon lepton number (or more) }\end{array}$ & $\begin{array}{l}\text { Two (or more) nonsequential, produced } \\
\text { in } e^{+} e^{-} \text {annihilation and neutrino } \\
\text { scattering }\end{array}$ & $\begin{array}{l}\text { Two (or more), produced by ordinary } \\
\text { neutrino scattering }\end{array}$ \\
\hline
\end{tabular}

tinguish different models. ${ }^{15}$ Future experiments may soon narrow down the alternative possibilities In the framework of gauge theories, "quark-lepton symmetry" has been conjectured-if leptons and quarks can be embedded in a unified framework. If this conjecture makes sense, the lepton spectrum may shed light on the quark spectrum. Another interesting and yet unsolved problem is the $m_{e} / m_{\mu}$ mass ratio. Attempts in the past to calculate the $m_{e} / m_{\mu}$ mass ratio have failed in simple renormalizable models. It would give us hope if the actual lepton spectrum were to become known. It may be meaningful to talk about this "symmetry of the leptons" if their spectrum can be experimentally established.

Finally, we like to comment on the magnitudes of neutral currents. At present, no data on the various processes (in Sec. II) which can test the lepton number of the heavy lepton are available. No events of the kind (1)-(3) of Sec. II are reported ${ }^{1}$ which are inconsistent with being background. But one notes that the SLAC-LBL data have uncertainties in hadron-lepton identification and (thus) in the estimate of the background. The fact that no large cross sections of neutral-current-induced processes have been seen can be interpreted as an indication that the neutral-current contribution is small. Even if it is small, it is important to look for the signals we have suggested. A few clean events of processes (1)-(3) in Sec. II would establish the nonsequential nature of the heavy lepton and the existence of neutral currents coupling to $L^{-}$and $e^{-}$.

The present data taken at face value gives the following limit on the neutral-current contribution:

(1) The "anomalous" cross section for $\mu^{*} \mu^{-}$, $\mu^{+} e^{-}+e^{+} \mu^{-}$, and $e^{+} e^{-}$being consistent with the ratio $1: 2: 1$ would suggest that the branching ratios for $B\left(L^{-} \rightarrow \mu^{-}+\bar{\nu}_{\mu}+\nu_{L}\right)$ and $B\left(L^{-} \rightarrow e^{-}+\bar{\nu}_{e}+\nu_{L}\right)$ are approximately equal. From (3.25) and (3.26), one would conclude that

$$
\delta \beta^{2}<1 \text {. }
$$

(2) Four-charged-lepton production with all lepton momenta $\geqslant 0.65 \mathrm{GeV}$ is not seen at a comparable rate as the $\mu^{\mp} e^{ \pm}$cross section. But one notes that most of the leptons are restricted by phase space and will not satisfy the above momentum criteria. A careful search for the fourcharged-lepton events is needed. Lack of such events would suggest from (3.27) and (3.28) that

$$
\delta \gamma<1 \text {. }
$$

(3) The cross section of $\mu^{ \pm} e^{F}$ events assuming a one-photon cross section for charged-heavylepton pair production gives the following branching ratio ${ }^{16}$ :

$$
\left[\frac{\Gamma\left(L^{-}-e^{-}\left(\mu^{-}\right)+\nu_{L}+\bar{\nu}_{e(\mu)}\right)}{\Gamma_{\text {total }}}\right]_{\exp } \simeq 0.17 \text {. }
$$

If only charged currents contribute, as in the case of the sequential heavy leptons, one finds by theoretically estimating the semileptonic decay rates that ${ }^{17}$

$$
\frac{\Gamma\left(L^{-}-e^{-}\left(\mu^{-}\right)+\nu_{L}+\bar{\nu}_{e(\mu)}\right)}{\Gamma_{\text {total }}} \sim 0.18 .
$$

For nonsequential charged-heavy-lepton decay one concludes from (3.25) and (3.26) with the aid of (4.1)-(4.3) that $\delta \gamma / x$ cannot be large. Because of the uncertainties in the present data as well as in theoretical estimates definite conclusions cannot at present be drawn.

\section{ACKNOWLEDGMENTS}

We thank H. Joos, G. Kramer, and T. Walsh for reading the manuscript, and $\mathrm{T}$. Walsh and $\mathrm{K}$. Fujikawa for several useful comments. One of us (A.A.) acknowledges the financial support of the Alexander von Humboldt Foundation. 
${ }^{1}$ M. L. Perl et al., Phys. Rev. Lett. $\underline{35}, 1489$ (1975). ${ }^{2}$ C. H. Albright, Phys. Rev. D 13, 2508 (1976).

${ }^{3}$ B. Barish et al., Phys. Rev. Lett. 32, 1387 (1974). ${ }^{4}$ Charged heavy leptons carrying electronic lepton number could be produced in deep-inelastic scattering, but since they are suppressed by $G_{F}{ }^{2}$, they are very difficult to produce.

${ }^{5}$ We assume two-component neutrinos for our discussion. If neutrinos are four-component objects and the right-handed components couple to charged heavy leptons, they can effectively be treated as the sequential case. (For massless four-component neutrinos, one can as well call the right-handed component a new kind of neutrino.)

${ }^{6}$ Y. S. Tsai, Phys. Rev. D 4, 2821 (1971); J. D. Bjorken and C. H. Llewellyn Smith, ibid. $\underline{7}, 887$ (1973). See M. L. Perl and P. Rapidis, SLAC Report No. SLACPUB-1496, 1974 (unpublished) for more references.

${ }^{7} \mathrm{~W}$ e assume that neutral currents do not couple to leptons of different lepton numbers as in most gaugetheory models.

${ }^{8}$ This mechanism to distinguish sequential charged heavy leptons from the nonsequential and new hadron decays has been pointed out by T. C. Yang, Univ. of Maryland Tech. Report No. 76-025 (unpublished).

${ }^{9}$ T. Das, V. S. Mathur, and S. Okubu, Phys. Rev. Lett. $\underline{18}, 761$ (1967).
${ }^{10}$ T. Das, V. S. Mathur, and S. Okubu, Phys. Rev. Lett. 19,470 (1967).

${ }_{11}^{11}$ J. J. Sakurai and R. J. Oakes, Phys. Rev. Lett. 19 , 1266 (1967).

${ }^{12} \mathrm{~S}$ ee, for example, the review of B. W. Lee, Fermilab Report No. 75/72 (unpublished).

${ }^{13}$ T. C. Yang, Ref. 8 and Phys. Rev. D 13, 1322 (1976); B. Stech, Heidelberg report (unpublished).

${ }^{14} \mathrm{~J}$. Schechter and Y. Ueda, Phys. Rev. D 8, 484 (1973);

J. Schechter and M. Singer, ibid. 9,1769 (1974);

L. Clavelli and T. C. Yang, ibid. 10, 658 (1974),

V. Gupta and H. S. Mani; ibid. 10, 1310 (1974), G. G. Ross, report (unpublished); M. Yoshimura, Tohokon University Report No. 75/131, 1975 (unpubli shed).

${ }^{15}$ Experimental tests of neutral-heavy-lepton production versus new hadron production in neutrino scattering have been discussed by T. C. Yang, DESY Report No. 76/03 (unpublished).

${ }^{16}$ M. L. Perl, lectures given at McGill University summer school; 1975 SLAC Report No. SLAC-PUB-1592 (unpublished).

${ }^{17}$ The number quoted here is taken from Tsai's paper after correcting the hadronic continuum contribution. However, these numbers are only a rough estimate because of the various approximations used to evaluate the decays. 